\title{
Long-term results of maintenance of lacrimal silicone stent in patients with functional epiphora after external dacryocystorhinostomy
}

\author{
Min Joung Lee ${ }^{1}$ Jinku Park ${ }^{2} \cdot$ Min Kyu Yang ${ }^{3}$ Youn Joo Choi $\mathbb{C}^{4} \cdot$ Namju Kim $^{5} \cdot$ Ho-Kyung Choung ${ }^{6,7}$. \\ Sang In Khwarg $\mathbb{1}^{3,7}$
}

Received: 15 March 2019 / Revised: 25 July 2019 / Accepted: 13 August 2019 / Published online: 16 September 2019

(c) The Author(s), under exclusive licence to The Royal College of Ophthalmologists 2019

\begin{abstract}
Purpose To evaluate long-term outcomes of maintenance of lacrimal silicone stent for the management of functional epiphora after anatomically patent external dacryocystorhinostomy (DCR).

Methods We retrospectively reviewed the medical records of 101 eyes of 75 patients who were diagnosed to have functional epiphora after external DCR from 2005 to 2014. Functional epiphora was defined as epiphora that persisted or recurred even after patent DCR confirmed by a lacrimal irrigation test. Secondary silicone intubation was indicated when the patients wanted a further intervention. The stent was intended to be kept in situ unless there was a stent-related complication or the patient wanted removal.

Results In total, 34 of 75 patients $(45.3 \%, 52$ eyes) who agreed to the intervention underwent secondary silicone intubation. The success rates at 1,3 , and 5 years after surgery were $96.2 \%, 75.5 \%$, and $70.2 \%$, respectively. At the final follow-up (mean $72.7 \pm 26.4$ months), $32(61.5 \%)$ eyes chose to retain the silicone tube: silicone stent was well maintained without epiphora and complications once inserted in 18 eyes (34.6\%), whereas tube replacement was needed in 14 eyes $(26.9 \%)$ because of nasal crust or whitish plaque formation on the tube surface. In 13 cases (25.0\%), silicone stent was removed because of tube-related complications, and the most common complication was canaliculitis $(n=8,15.4 \%)$.

Conclusions Secondary intubation and maintenance of the stent is an effective and simple procedure for functional epiphora. The main obstacle to long-term maintenance is tube-associated canaliculitis.
\end{abstract}

\section{Introduction}

Sang In Khwarg

khwarg@snu.ac.kr

1 Department of Ophthalmology, Hallym University Sacred Heart Hospital, Anyang, Korea

2 Barun Seoul Eye Clinic, Seongnam, Korea

3 Department of Ophthalmology Seoul National University Hospital, Seoul, Korea

4 Department of Ophthalmology, Kangdong Sacred Heart Hospital, Hallym University Medical Center, Seoul, Korea

5 Department of Ophthalmology, Seoul National University Bundang Hospital, Seongnam, Korea

6 Department of Ophthalmology, Boramae Medical Center, Seoul, Korea

7 Department of Ophthalmology, College of Medicine, Seoul National University, Seoul, Korea
Dacryocystorhinostomy (DCR) is a well-established treatment option for most cases of lacrimal drainage obstruction, allowing direct drainage of tears via a surgically created bypass between the lacrimal sac and nasal cavity. The success rates for DCR have been reported to be very high at $70-90 \%$, but literature reviews have shown that there is a discrepancy between objective success rate assessed by anatomical patency and symptomatic success [1, 2]. In some cases, patients complain of bothersome epiphora although the DCR site is patent and clinical examination is unremarkable otherwise, and this condition is so-called "functional epiphora" after patent DCR [3-5].

The treatment of functional epiphora is challenging and various surgical procedures have been tried, including secondary silicone tube intubation, lid-tightening procedures, punctoplasty and conjunctivo-DCR with Jones tube [5]. Our group has used secondary silicone tube intubation to 
manage functional epiphora after external DCR and reported good early results [6]. Epiphora was resolved after the procedure in all 13 patients with a mean follow-up period of 13.8 months. However, the stent is usually intended to be kept in situ permanently, so the long-term results are very important. In this study, we investigated the long-term success rate and complications of the secondary silicone intubation procedure for patients with functional epiphora after anatomically patent external DCR.

\section{Methods}

We reviewed the medical records of patients who were diagnosed as functional epiphora after external DCR performed by a single surgeon (SIK) in Seoul National University Hospital Oculoplasty Clinic from 2005 to 2014. External DCR operations were indicated for complete nasolacrimal duct obstruction, common canalicular obstruction, and distal canalicular obstruction. The external DCRs were performed using a standardised procedure described in our previous reports $[3,7,8]$. In most instances, a silicone bicanalicular tube (solid tube, 0.64-mm diameter, C-Line canalicular intubation set 8590450; Medtronic Ophthalmics, Jacksonville, FL, USA) was inserted. Double silicone intubation was performed in cases with common canalicular or distal canalicular obstruction [7]. Sometimes, we did not insert the stent in patients with large lacrimal sac and intact canalicular system [8]. All patients were followed up after 1 week, 1 month, and between 4 and 6 months postoperatively, and then at variable intervals thereafter. The silicone tube was removed 4-6 months after surgery.

Functional epiphora after DCR was defined as; (1) recurrent or persistent epiphora after DCR, (2) symptomatic epiphora with Munk score $\geq 2$, (3) anatomically patent DCR confirmed with patent irrigation without significant reflux on a syringing test with or without a fluorescein dye disappearance test. Lacrimal probing was also performed through the upper and lower puncta to exclude partial stricture of lacrimal passage and to predict the feasibility of silicone intubation with minimal anaesthesia. Lacrimal patency was confirmed when the probe was easily introduced into the deep nasal cavity and the end of the probe was seen in the nasal cavity. Patients with facial nerve palsy, eyelid abnormalities including ectropion, entropion, significant eyelid laxity, or secondary lacrimal drainage obstruction were excluded. For patients with functional epiphora after DCR, details of the procedure of secondary bicanalicular silicone intubation which would be intended to maintain the stent as long as possible unless complicated, were explained to the patients. If patients agreed on further treatment, lacrimal silicone intubation through the rhinostomy was performed at the outpatient clinic [6].

At the beginning of the procedure, the nasal cavity was packed with a gauge soaked in a 1:1 mixture of epinephrine $(1 \mathrm{mg} / \mathrm{ml})$ and $4 \%$ lidocaine in patients with a narrow nasal cavity, whereas $10 \%$ lidocaine solution (Beracaine ${ }^{\circ}$; Firson inc, Cheonan, Korea) was just sprayed into the nostril in cases who had a wide nasal cavity. A bicanalicular silicone tube was introduced into the DCR rhinostomy site through the upper and lower punctum and drawn out from the nasal cavity with bayonet forceps or straight haemostat under direct visualisation. The ends of the tube were tied together with 6-0 black silk at a point where the knot could be seated deeply in the nasal cavity and then left free in the nasal cavity without any fixation. We intended to keep silicone stents on a permanent basis: as long as possible after reintubation.

If epiphora was sufficiently relieved at the first visit after re-intubation, we recommended the patients to keep the stent and to attend follow-up sessions regularly every 6 months. In addition, we instructed the patients to visit earlier than the schedule, if any discomfort occurred between follow-up visits. On every visit, patients were asked about their epiphora symptom and examined for tear meniscus height, lacrimal punctum, and status of the silicone tube.

The main outcomes of this study were (1) onset of functional epiphora, (2) long-term success rate of the secondary silicone intubation, and (3) stent-associated complications of this management. The onset of functional epiphora was classified as persistent epiphora, early recurrence, and delayed recurrence. Persistent epiphora was defined when tearing symptom persisted after external DCR without silicone tube. Early recurrence was deemed to occur when the epiphora recurred within 6 weeks after silicone tube removal. Delayed recurrence was defined as epiphora that recurred more than 6 weeks after tube removal. Postoperative success was defined as retention of stent with improved epiphora symptom and without significant discomfort or complication. Subjective improvement of epiphora was considered when equivalent to Munk's score 0 or 1. Fluorescein dye test was not routinely conducted in all patients. This study followed the tenets of the Declaration of Helsinki. The protocol of this study was approved by the institutional review board of Seoul National University Hospital (1608-124-787). The requirement to obtain informed consent was waived because of the retrospective nature of this study and the anonymization of all data. The Kaplan-Meier survival analysis was used to calculate cumulative long-term success rate of secondary silicone intubation until the removal of the tube. Statistical analyses were performed using GraphPad Prism 7.0 (GraphPad Software, La Jolla, CA, USA). 


\section{Results}

During the enrolment period, 101 eyes of 75 patients were considered to have functional epiphora even after anatomically patent external DCRs. All patients complained of persistent or recurrent epiphora symptom even though the DCR site was patent by the syringe irrigation test. Mean age of the patients was $56.9 \pm 10.6$ years. Fourteen patients $(18.7 \%)$ were male and $61(81.3 \%)$ were female. Laterality included $58(57.4 \%)$ right eyes and $43(42.6 \%)$ left eyes. Most of the patients (70 of 101 eyes, 69.3\%) complained of recurrence of epiphora early ( $\leq 6$ weeks) after the removal of the silicone tube. Twenty-two eyes $(21.8 \%)$ presented delayed epiphora more than 6 weeks after tube removal. Nine eyes $(8.9 \%)$ that manifested continuous tearing symptom after external DCR without silicone tube were classified as having persistent epiphora. The timing of epiphora, the number of patients who received the secondary silicone intubation and the management outcomes are shown in Fig. 1.

Overall, 34 of 75 patients (45.3\%, 52 eyes) agreed to active intervention for the functional epiphora and received the secondary silicone tube intubation. Before the procedure, all patients were confirmed to have a patent lacrimal system by syringing test and lacrimal probing test, and not to have any condition that indicated exclusion. There was no significant intraoperative complication. Postoperatively, 50 of 52 (96\%) eyes demonstrated immediate improvement of epiphora with the silicone tube in place and presented a normal tear meniscus height by slit lamp biomicroscopic examination. The remaining two eyes $(4 \%)$ stated that epiphora symptom was partly improved but not completely resolved. We also analysed the success rate of the secondary silicone intubation procedure in terms of tube maintenance probability using the Kaplan-Meier survival curve (Fig. 2). The postoperative success rate was $96.2 \%$ at 1 year, $75.5 \%$ at 3 years, and $70.2 \%$ at 5 years. The median time from secondary silicone intubation to removal was 6.6 years.

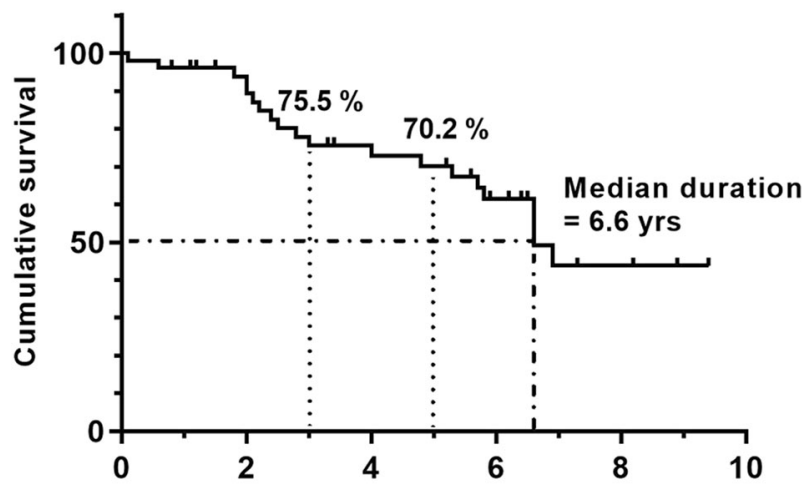

Time after secondary silicone intubation (yrs)

Fig. 2 A survival curve for the maintenance of the stent after secondary silicone intubation for functional epiphora after external DCR
Fig. 1 Flow diagram of the study population

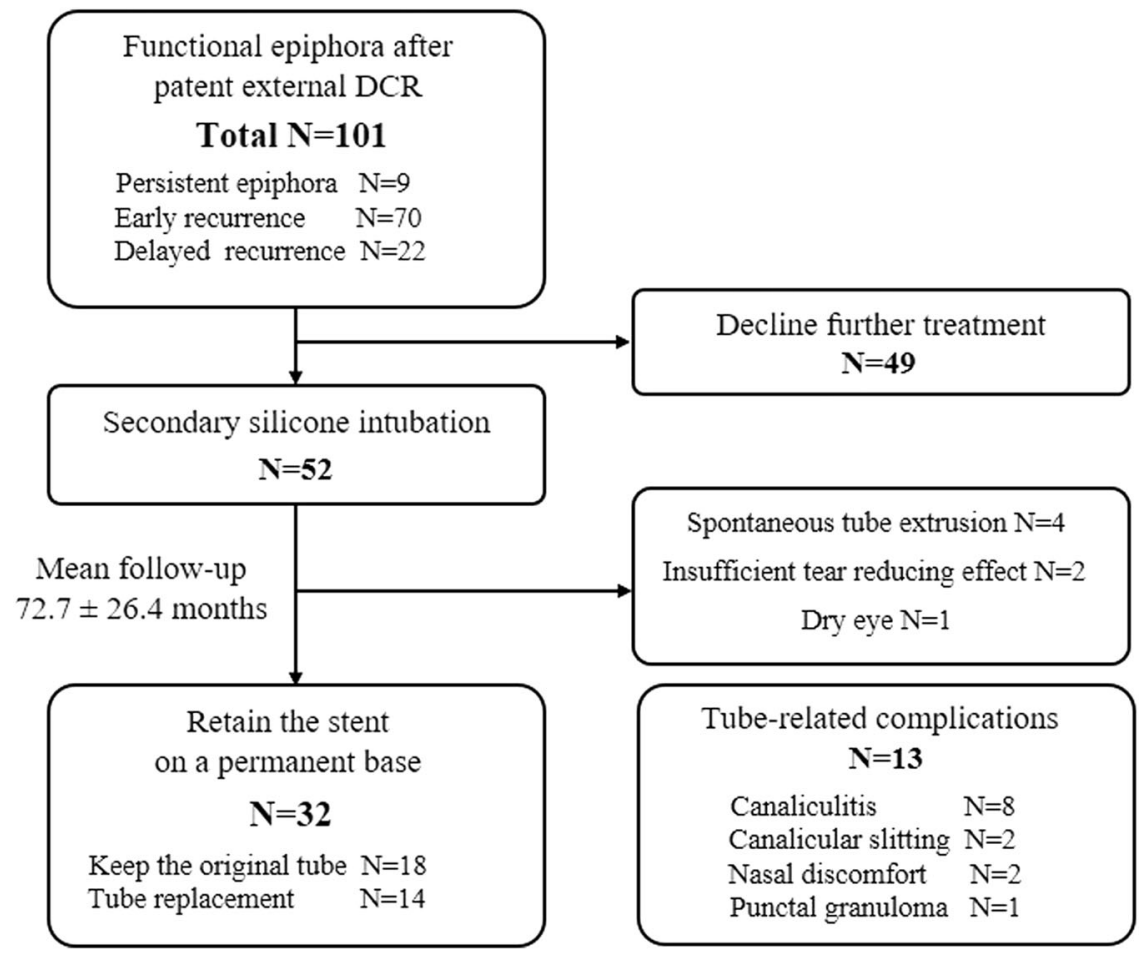


Fig. 3 Representative cases with complications associated with permanent-base secondary silicone tube intubation. a Whitish plaque on the stent surface, $\mathbf{b}$ canaliculitis with mild punctal slitting, $\mathbf{c}$ punctal slitting, d punctal granuloma

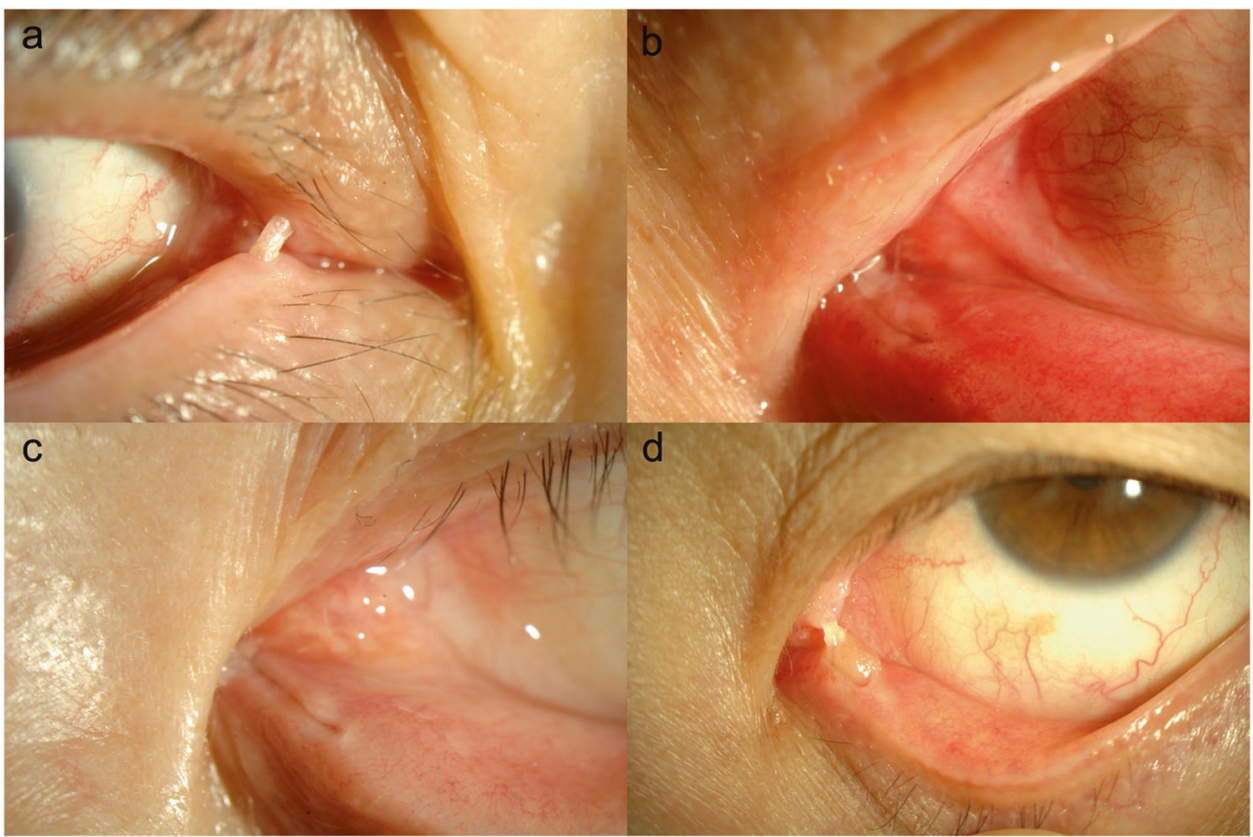

At a mean follow-up period of $72.7 \pm 26.4$ months after silicone intubation, 32 of $52(61.5 \%)$ eyes retained the silicone stent on a permanent basis. In 18 (34.6\%) eyes, the epiphora was completely resolved without any significant discomfort or complications, and the stent was well maintained after one intubation procedure. Two patients experienced lateral displacement and prolapse of the stent. However, it was possible to easily reposition the stent by just retracting the tube at the nasal side. In 14 (26.9\%) eyes, the epiphora was significantly reduced but we recommended stent replacement because of the formation of a nasal crust or whitish plaque along the stent surface, which carries a risk of canalicular slitting or canaliculitis (Fig. 3a). The numbers of exchange procedures were: one in seven eyes, two in five eyes, four in one eye, and six in one eye. The mean time from the secondary silicone intubation to the first exchange of the stent was $42.08 \pm$ 22.53 months.

In the remaining 20 cases (38.4\%), silicone tubes were not maintained or removed during the follow-up period. In four patients, the silicone tube was lost spontaneously. Two patients wanted the tube removed because of insufficient relief from epiphora. There was one patient who wanted the stent removed because of the dry eye symptom. Thirteen patients $(25 \%)$ wanted the stent removed because of stentrelated complications or discomfort as follows: canaliculitis ( $n=8$, Fig. 3b), canalicular slitting $(n=2$, Fig. 3c), nasal discomfort $(n=2)$, and punctal granuloma $(n=1$, Fig. 3 d $)$. Among these cases, a third intubation was performed at a later date in six eyes after the resolution of the complications.

\section{Discussion}

In the present study, functional epiphora usually occurred shortly after the removal of the silicone tube, which had been primarily inserted during external DCR. Most of the patients (70 of 101 eyes, 69.3\%) complained recurrence of epiphora early ( $\leq 6$ weeks) after the removal of the silicone tube. This finding clinically implied that lacrimal surgeons should check up the epiphora symptom of the patients for a period after the removal of the stent. This finding can also be the rationale behind the re-intubation procedure of silicone stent for the management of functional epiphora. Nine eyes (8.9\%) were classified as having persistent epiphora. They all received external DCRs without silicone intubation because they had a large lacrimal sac and a wide nasal cavity [8], but they complained of continuous epiphora even with patent rhinostomy. These cases also supported the role of lacrimal stent facilitating tear drainage. The pathophysiologic causes underlying functional epiphora are unclear. Some authors commented that defective action of the lacrimal pump might be the main cause of functional epiphora $[9,10]$. We hypothesised that the stent could enhance the lacrimal pump function by helping the apposition of upper and lower puncta and increasing the capillarity of the lacrimal drainage pathway [6].

After the secondary silicone tube intubation, most patients $(50$ of 52 eyes $)$ experienced immediate improvement of epiphora, and in the long term, $61.5 \%$ of the patients were able to retain the silicone tube during a mean follow-up period of 72 months. Some patients could keep the stent persistently once inserted, but other 
patients needed replacement of the stent because of the formation of a nasal crust or plaque formation along the stent surface. These patients were satisfied with the effect of the stent in terms of reducing epiphora and wanted to retain the stent despite the procedure of replacement. Stent replacement was recommended when the whitish plaque coating the tube surface was prominent. We considered the plaque coating along the stent as a precursor of canaliculitis. A large nasal crust was another main reason for stent replacement. Significant nasal crusts not only caused foreign body or tickling sensation in the nasal cavity, but also punctal and canalicular slitting by pulling the stent to the nasal side. When the stent was drawn to the medial canthus or there was a punctal slitting, we examined the nasal cavity and tried to remove nasal crusts. If it was not easy to remove the crusts, we recommended stent replacement.

The main reason for the removal of the silicone tube was tube-related complication itself, rather than insufficient efficacy of the tube. The most common complication was tube-related canaliculitis. Canaliculitis is characterised by punctal discharge, punctal or canalicular swelling and erythema [11-13]. Although the most common form is the primary canaliculitis without any identifiable cause, canaliculitis can occur secondarily after punctal plug or silicone tube insertion. Punctal pluginduced canaliculitis has been well-documented to have a mild clinical course and be associated with Pseudomonas, and the literature recommends plug removal $[14,15]$.

With regard to silicone tube-associated canaliculitis, biofilm formation on the surface plays an important role [16-19]. Biofilm is an assembly of surface-associated microbial cells that is enclosed in an extracellular polymeric substance matrix. Biofilm reduces the metabolic needs of bacteria and increases the resistance to antibiotics, resulting in enhanced survival of the bacteria. Although a silicone tube is a relatively safe synthetic material, biofilm can be formed on any surface. Moreover, a silicone tube is always exposed to microbial flora of the nasal cavity. A specific pathogen species can be associated with stent-related canaliculitis, but we did not perform a routine culture of the stents. Samimi et al. [19] compared the microbiology culture results between infected stent with a non-infected stent and reported Gram-positive organisms were dominant in cultures of non-infected stents whereas non-tuberculous Мусоbacterium was the predominant organism found in infected stents. Kim et al. [17] cultured silicone stents removed after DCR surgery and reported that Pseudomonas infection was significantly associated with surgical failure.

The definitive treatment option for stent-related canaliculitis is removal of the stent. In this study, the signs and symptoms of canaliculitis were all resolved within 1 week after stent removal in all patients. Some of those patients wanted re-insertion of the stent, and hence received the third silicone intubation after a complete resolution of inflammation and sufficient rest period. The results of this study suggested that the technical enhancement of the stent, such as novel material chemistry or surface coating, would be helpful for the long-term maintenance of the stent. Future studies developing more biocompatible lacrimal stent will be needed.

The strength of this study is the uniformity in the management strategy for the patients with functional epiphora after DCR and long-term results of permanent silicone stent intubation. However, this study has several weaknesses. First, this study had a retrospective design and a small number of cases without a control group, hampering the inference of concrete conclusions. Second, we only used the lacrimal syringing test to assess anatomical patency of DCR and we did not perform fluorescein dye disappearance test or nasal endoscopic examination of rhinostomy in all cases. Thus, some cases of this study possibly had stenosis of rhinostomy. However, we examined the patency by inserting the lacrimal probe through the rhinostomy, the probe was easily inserted to the nasal cavity, and the end of the probe was visible in all cases. Third, we did not consider lid-tightening procedure for any of the patients, although we excluded patients who had significant eyelid laxity. Shams et al. [5] reported that eyelid tightening procedure was effective in $50 \%$ of cases of functional epiphora, and even they had a lesser degree of eyelid laxity. Finally, we did not perform a microbiologic culture of the removed stent which may have provided helpful information about the causative pathogen.

In conclusion, post-DCR epiphora mostly recurred shortly after removal of the stent, suggesting the practice of sustained monitoring of epiphora. Secondary intubation of the silicone tube was an effective treatment option for functional epiphora after DCR. However, the long-term benefit of this procedure was limited by the possible need for tube replacement and a considerable frequency of complications. Tube-associated canaliculitis was the main complication leading to failure and should be solved to maintain the stent permanently. Further research into tuberelated canaliculitis may aid in the development of useful prevention strategies.

\section{Summary table}

\section{What was known before?}

- Some patients experience persistent or recurred epiphora even after anatomically patent 
dacryocystorhinostomy: so-called 'functional epiphora after dacryocystorhinostomy'.

- Silicone intubation is one of the treatment options for functional epiphora, but the long-term results are still ambiguous.

\section{What this study adds?}

- Secondary silicone intubation was effective in $61.5 \%$ of patients with a mean follow-up period of 72 months.

- The main reason for the removal of the silicone stent was tube-related complication, and the most common complication was canaliculitis.

\section{Compliance with ethical standards}

Conflict of interest The authors declare that they have no conflict of interest.

Publisher's note Springer Nature remains neutral with regard to jurisdictional claims in published maps and institutional affiliations.

\section{References}

1. Beshay N, Ghabrial R. Anatomical and subjective success rates of endonasal dacryocystorhinostomy over a 7-year period. Eye. 2016;30:1458-61.

2. Coumou AD, Genders SW, Smid TM, Saeed P. Endoscopic dacryocystorhinostomy: long-term experience and outcomes. Acta Ophthalmol. 2016;95:74-8.

3. Lee MJ, Khwarg SI, Kim IH, Choi JH, Choi YJ, Kim N et al. Surgical outcomes of external dacryocystorhinostomy and risk factors for functional failure: a 10-year experience. Eye. 2017;31:691-7.

4. Sahlin S, Rose GE. Lacrimal drainage capacity and symptomatic improvement after dacryocystorhinostomy in adults presenting with patent lacrimal drainage systems. Orbit. 2001;20:173-9.

5. Shams PN, Chen PG, Wormald PJ, Sloan B, Wilcsek G, McNab A, et al. Management of functional epiphora in patients with an anatomically patent dacryocystorhinostomy. JAMA Ophthalmol. 2014;132:1127-32.
6. Kim NJ, Kim JH, Hwang SW, Choung HK, Lee YJ, Khwarg SI. Lacrimal silicone intubation for anatomically successful but functionally failed external dacryocystorhinostomy. Korean $\mathbf{J}$ Ophthalmol. 2007;21:70-73.

7. Hwang SW, Khwarg SI, Kim JH, Choung HK, Kim NJ. Bicanalicular double silicone intubation in external dacryocystorhinostomy and canaliculoplasty for distal canalicular obstruction. Acta Ophthalmol. 2009;87:438-42.

8. Choung HK, Khwarg SI. Selective non-intubation of a silicone tube in external dacryocystorhinostomy. Acta Ophthalmol Scand. 2007;85:329-32.

9. Delaney YM, Khooshabeh R. Fluorescein transit test time and symptomatic outcomes after external dacryocystorhinostomy. Ophthalmic Plast Reconstr Surg. 2002;18:281-4.

10. Detorakis ET, Drakonaki E, Papadaki E, Pallikaris IG, Tsilimbaris MK. Watery eye following patent external DCR: an MR dacryocystography study. Orbit. 2010;29:239-43.

11. Kaliki S, Ali MJ, Honavar SG, Chandrasekhar G, Naik MN. Primary canaliculitis: clinical features, microbiological profile, and management outcome. Ophthalmic Plast Reconstr Surg. 2012; 28:355-60.

12. Lee MJ, Choung HK, Kim NJ, Khwarg SI. One-snip punctoplasty and canalicular curettage through the punctum: a minimally invasive surgical procedure for primary canaliculitis. Ophthalmology. 2009;116:2027-.e2022.

13. Zaldivar RA, Bradley EA. Primary canaliculitis. Ophthalmic Plast Reconstr Surg. 2009;25:481-4.

14. Freedman JR, Markert MS, Cohen AJ. Primary and secondary lacrimal canaliculitis: a review of literature. Surv Ophthalmol. 2011;56:336-47.

15. Huang YY, Yu WK, Tsai CC, Kao SC, Kau HC, Liu CJ. Clinical features, microbiological profiles and treatment outcome of lacrimal plug-related canaliculitis compared with those of primary canaliculitis. Br J Ophthalmol. 2016;100:1285-9.

16. Kim DJ, Park JH, Chang M. Species-specific characteristics of the biofilm generated in silicone tube: an in vitro study. BMC Ophthalmol. 2018;18:85.

17. Kim SE, Lee SJ, Lee SY, Yoon JS. Clinical significance of microbial growth on the surfaces of silicone tubes removed from dacryocystorhinostomy patients. Am J Ophthalmol. 2012;153: 253-.e251.

18. Kim SH, Park CY, Hwang SW, Chang M. Clinical significance of biofilm on silicone tubes removed from patients with nasolacrimal duct stenosis. J Craniofac Surg. 2018;29:462-5.

19. Samimi DB, Ediriwickrema LS, Bielory BP, Miller D, Lee W, Johnson TE. Microbiology and biofilm trends of silicone lacrimal implants: comparing infected versus routinely removed stents. Ophthalmic Plast Reconstr Surg. 2016;32: $452-7$. 